\title{
Modelling and interaction of needle domains in Barium Titanate single crystals
}

\author{
Dan $\mathrm{Sui}^{a}$, John E. Huber ${ }^{b *}$ \\ ${ }^{a}$ School of Aeronautics, Northwestern Polytechnical University, Xi'an, Shaanxi, 710072, \\ China \\ ${ }^{b}$ Department of Engineering Science, University of Oxford, Parks Road, Oxford, OX1 3PJ, \\ $U K$
}

\begin{abstract}
Needle domains are thin, lamellar domains that can appear as fine lines or stripes in a ferroelectric crystal. They affect the poling, switching, and other properties of ferroelectrics. A model is established to study the stress and electric fields caused by needle domains and their interaction. Considering the electrical and mechanical compatibility conditions at the tip of a needle domain, the fields are represented using equivalent edge dislocations and line charges. Accordingly, the dislocation fields derived by Barnett and Lothe for anisotropic piezoelectric media are employed. A modified Peach-Koehler force and calculations of the total energy due to the needle domains are used to study the formation, interaction and stability of needle patterns, taking full account of the strong anisotropy and electromechanical coupling present.

The interaction of pairs of needle domains in an infinite piezoelectric with properties of barium titanate is found to be dominated by the electrostatic terms. This makes comb-like arrays of needle domains unstable if perfectly insulating properties are assumed. By considering redistribution of charge, stable equilibrium states for arrays of needle domains are found; these agree well with experimental observations. The model explains the stability of various observed needle patterns and also how unstable patterns evolve.
\end{abstract}

Keywords: ferroelectric, domain, barium titanate, dislocation

*Corresponding author. email: john.huber@eng.ox.ac.uk

Preprint submitted to European Journal of Mechanics-A/Solids

November 26, 2019 
dynamics

\section{Introduction}

Ferroelectric crystals commonly contain thin, lamellar domains that terminate within the crystal. Such domains appear as fine lines, or "needles" that can change in length when the crystal is subjected to stress or electrical loading. The 5 resulting arrangements of needle domains are significant for determining the poling, switching and piezoelectric behaviour of the crystals 11. The appearance and rearrangement of needle domains is also associated with crack propagation [2] and can influence material toughness [3, 4]. $\mathrm{BaTiO}_{3}$ single crystals, as lead-free ferroelectric materials, have been widely used to investigate ferroelectric behaviour [5, 6, 7, 8. One reason is that they are advantageous in theoretical work, due to the relative simplicity of their crystal structure, when compared to other ferroelectrics. The material also offers the practical advantages of providing high polarization and ferroelectric strain, along with excellent electromechanical properties. Perfect single crystals with an ideal single domain structure are hard to manufacture and instead needle-shaped domains are commonly found in the crystals [6, 9]. Therefore, an understanding of the interaction and evolution of needle domain structures is desirable.

Several researchers have investigated the behaviour of needle-shaped domains through theoretical and experimental methods [6, 10, 11, 12, 13. In early work, Forsbergh [6] observed $90^{\circ}$ a-c and a-a needle domains in tetragonal $\mathrm{BaTiO}_{3}$ single crystals. Bornarel et al. [11, 12, 13 investigated the interaction and evolution of needle domains in ferroelectric $\mathrm{KH}_{2} \mathrm{PO}_{4}$ theoretically and experimentally. Evidence was found for the existence of long-range interactions, up to $200 \mu \mathrm{m}$ between the domains, and a model of the needle domain tip as an assembly of edge dislocations was developed. Accordingly, the fields due to a classical edge dislocation in an isotropic elastic solid were used for interpretation of experimental observations.

Salje and Bueble et al. [14, 15, 16, 10, 17] researched needle domains in 
$\mathrm{LaAlO}_{3}$ and $\mathrm{SrTiO}_{3}$, which are analogs of the $(\mathrm{Me}, \mathrm{Fe}) \mathrm{SiO}_{3}$ perovskite thought to be a major component of the Earth's lower mantle. They argued that nearby domain wall corners are attracted together, forming needle domains with sharp tips. Little [18 studied needle domains with typical widths of $10-30 \mu m$ in tetragonal $\mathrm{BaTiO}_{3}$, finding that they nucleated at the crystal boundaries at a critical field value. After that, the needle domains lengthened without broadenforced the needles back out of the crystal. Bornarel et al. 19 reported that the switching process in ferroelectric crystals is related to the twinning dislocations which are created at the tips of needle domains. Zhang et al. and Duan et al. observed $90^{\circ}$ a-a needle domains using transmission electron microscopy (TEM) 40 when they investigated the switching mechanism of multi-domains in $\mathrm{BaTiO}_{3}$ single crystal [20, 21, 22].

Comb-like patterns comprising many parallel needle domains terminating along the same line are commonly observed. Figure 1 shows such a comb pattern, with the needles terminating along a line approximately $45^{\circ}$ to the 45 crystallographic axes. This patterning has long been understood as an analogue of the formation of low angle boundaries from a set of dislocations; in this case each needle-tip is loosely analogous to a dislocation with a slip-plane parallel to the needle. Such a set of dislocations in an isotropic elastic solid tend to relax into a low energy configuration producing an array of dislocations lying ${ }_{50}$ in a plane normal to their slip planes. However, application of this analogy to $\mathrm{BaTiO}_{3}$ produces a puzzle: Firstly, the material is strongly anisotropic, and electromechanically coupled, so the equilibrium positions of dislocations should not be expected to correspond to those of an isotropic elastic medium. Secondly, there is a jump in polarization between the needle and its surroundings; the 55 resulting depolarization fields strongly affect the configuration and stability of domain needles [23]. Yet, in observations, the needle-tips commonly line up as if they behaved like dislocations in an isotropic elastic medium. To the authors' knowledge, this phenomenon has not been explained adequately up to now. Phase field or Ginzburg Landau models indicate that needle domains and arrays 


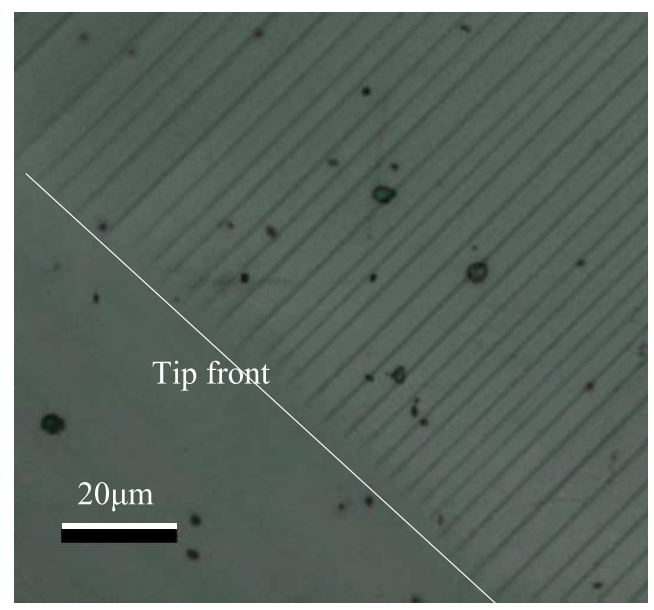

Figure 1: Comb-like needle domains in a $\mathrm{BaTiO}_{3}$ crystal, observed by optical microscopy. The crystallographic [100] and [010] directions lie parallel to the edges of the image. The needles are each a few $\mu \mathrm{m}$ across and their tips align at about $45^{\circ}$ to the crystallographic axes.

of needles in ferreoelectrics are typically unstable unless some special conditions such as compositional gradients or stabilizing constraints are present 24, 4, 25, 26. Thus there is a need to reassess the dislocation model in order to account for the observed patterning of needle domains.

In this paper, we focus on $90^{\circ}$ a-a needle domains and build a model to 65 simulate the interaction and evolution of needle domains in $\mathrm{BaTiO}_{3}$ single crystals, including the properties of crystalline anisotropy and electromechanical coupling. The fields of dislocations and line charges are used to represent the effect of a needle domain on the surrounding material. The Barnett and Lothe theory of a dislocation with a line charge is used and a modified Peach-Koehler force on needle domains is proposed to describe and explain their interaction.

\section{Modelling of needle domains}

At room temperature, $\mathrm{BaTiO}_{3}$ single crystals exhibit tetragonal symmetry (4mm), with lattice parameters $c$ and $a$ differing by $1.1 \%$. Typical configuration parameters and anisotropic material constants of tetragonal $\mathrm{BaTiO}_{3}$ single 
crystals with polarization direction $\left[\begin{array}{lll}0 & 1 & 0\end{array}\right]$ at room temperature are given in table 1.

Table 1: Material constants of $\mathrm{BaTiO}_{3}$ in tetragonal phase with polarization direction of [0 $10]$ [8, 27, 28

\begin{tabular}{ll}
\hline & $\mathrm{BaTiO}_{3}$ single crystal \\
\hline Structure & Tetragonal $(4 m m)$ \\
Lattice parameter, $a(\AA)$ & 3.992 \\
Lattice parameter ratio, $c / a$ & 1.011 \\
Curie temperature, $T_{c}\left({ }^{\circ} \mathrm{C}\right)$ & 120 \\
Spontaneous polarization, $p_{0}\left(\mathrm{C} / \mathrm{m}^{2}\right)$ & 0.26 \\
Spontaneous strain, $\varepsilon_{0}=2(c-a) / 3 a$ & 0.0073 \\
Elastic stiffness, $C(\mathrm{GPa})$ & $211\left(C_{11}\right), 114\left(C_{12}\right), 107\left(C_{13}\right), 160\left(C_{22}\right)$, \\
& $56.2\left(C_{44}\right), 127\left(C_{55}\right)$ \\
Piezoelectric coefficients, $e\left(\mathrm{C} / \mathrm{m}^{2}\right)$ & $32.6\left(e_{16}\right),-3.88\left(e_{21}\right), 5.48\left(e_{22}\right)$ \\
Dielectric constants, $\kappa$ & $1980\left(\kappa_{11} / \kappa_{0}\right), 48\left(\kappa_{22} / \kappa_{0}\right)$, \\
& $\kappa_{0}=8.854 \times 10^{-12}(\mathrm{~F} / \mathrm{m})$ \\
\hline
\end{tabular}

\subsection{Fields of dislocations and charges}

The formation of $90^{\circ}$ a-a type needle domains results from $90^{\circ}$ ferroelectric switching. Figure 2 illustrates the tip of one needle domain in a plane perpendicular to the $x_{3}$ axis. Here, the spontaneous polarization vectors of the parent domain and needle domain are $\mathbf{P}^{1}=\left[\begin{array}{lll}0 & 1 & 0\end{array}\right]^{T} p_{0}$ and $\mathbf{P}^{2}=\left[\begin{array}{lll}-1 & 0 & 0\end{array}\right]^{T} p_{0}$, respectively. The two sidewalls of this needle domain are $90^{\circ}$ domain walls, and satisfy the electrical and mechanical compatibility requirements for a stressfree and charge-free domain wall. However, the abrupt jumps in spontaneous 85 polarization and spontaneous strain near the needle domain tip do not satisfy compatibility conditions. Therefore one of two situations must occur: either some combination of dislocations and charges must be present to allow the mismatch in strain and polarization, or there must be equivalent fields (stress and 


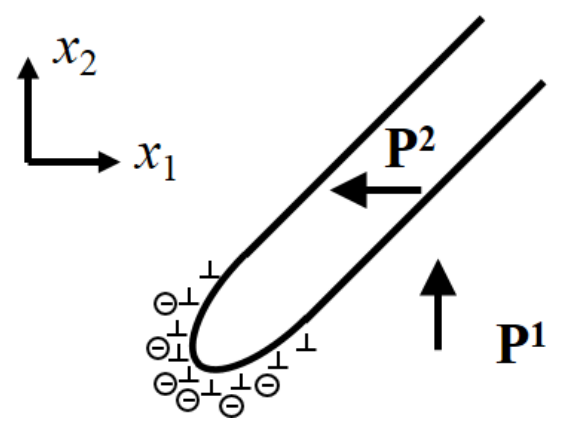

Figure 2: Distribution of effective dislocations and charge spread over the needle-tip due to the jump in spontaneous strain and electrical polarization.

electric fields) that modify the total strain and electric displacement away from the spontaneous strain and polarization state, enabling the compatibility conditions to be satisfied. Since $\mathrm{BaTiO}_{3}$ is a ceramic material and an insulator, there is little mobility of dislocations and charges. Thus it is likely that equivalent fields (stress and electric fields) are present in the vicinity of any microstructural features that entail abrupt jumps in spontaneous polarization and spontaneous strain.

An example of this is near the tip of a needle domain. The domain wall cannot adopt the prefectly compatible orientation based on the spontaneous polarization and strain alone; additional strain and electric displacement due to stresses and electric fields are required. The resulting distortion can be represented by a fictitious dislocation field. As shown in figure 3 each step in the $90^{\circ}$ domain wall produces a displacement $b_{0}$ parallel to the wall. The needletip strain fields can thus be approximated by those of an equivalent assembly of dislocations. Each equivalent dislocation has the Burgers vector $\mathbf{b}_{0}$, given by

$$
\mathbf{b}_{0}=\int_{c_{0}} d \mathbf{u}
$$

where, $c_{0}$ is a Burgers circuit surrounding the step in the wall, and $d \mathbf{u}$ represents the increment in displacement field resulting from the step in the domain wall.

Considering the thin width of needle domains, the concept of an 'effective 
(a)

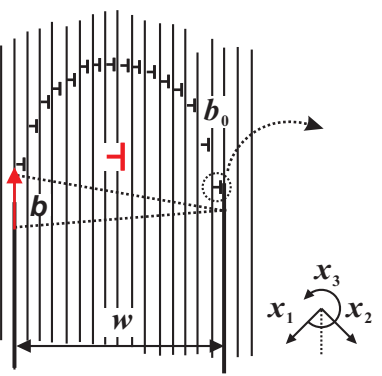

(b)

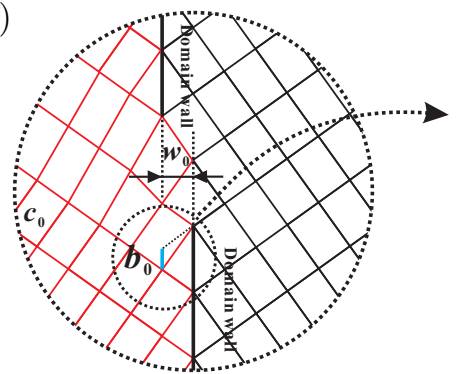

(c)

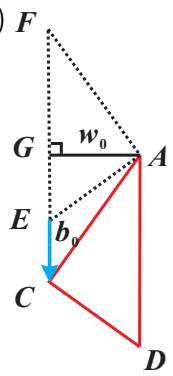

Figure 3: Schematic representation of the needle domain, (a) Needle-tip as an assembly of equivalent dislocations modified from Bornarel [12, (b) An equivalent dislocation in the $\mathrm{BaTiO}_{3}$ crystal lattice, (c) Geometry of unit cells used for calculating Burgers vector $\mathbf{b}_{0}$.

dislocation' with Burgers vector b can be adopted in order to estimate the field produced by all the equivalent dislocations assembled at the needle-tip. The far field around one needle domain tip can be approximated as that resulting from a single effective dislocation $\mathbf{b}$, with magnitude equal to the sum of all step dislocations $b_{0}$ through the whole width of needle domain:

$$
|\mathbf{b}|=\frac{w\left|\mathbf{b}_{0}\right|}{w_{0}} \approx 3 \varepsilon_{0} w
$$

where, $w$ is the width of the needle domain, and $\varepsilon_{0}$ is the spontaneous strain of the crystal lattice relative to the cubic state. The lateral step size $w_{0}$ can be expressed using the lattice parameters given in table 1 , and hence is related to $\varepsilon_{0}$.

As shown in figure 3(b), the crystal lattice on either side of the domain wall represents parent domain and needle domain. The corresponding polarization directions are approximately perpendicular to each other. In figure 3 (c), triangle-AFE shows the lattice position before switching, while triangle-ACD shows lattice position after $90^{\circ}$ switching. Due to $90^{\circ}$ domain switching, a slip displacement $b_{0}(\mathrm{EC})$ is created. Using the geometry in figure 3(c), the slip displacement $b_{0}$ and the lateral step size are given by 


$$
\begin{aligned}
w_{0} & =\frac{a c}{\sqrt{c^{2}+a^{2}}} \\
\left|\mathbf{b}_{0}\right| & =\frac{c^{2}-a^{2}}{\sqrt{c^{2}+a^{2}}}
\end{aligned}
$$

where, $a$ and $c$ are the lattice parameters of the $\mathrm{BaTiO}_{3}$ single crystal in the tetragonal phase. The lines $\mathrm{AF}$ and $\mathrm{AC}$ are equal to $c$, and the lines $\mathrm{AE}$ and $125 \mathrm{CD}$ are equal to $a$.

The representation of the needle-tip fields by those of a single effective dislocation is an approximation that neglects the inhomogeneity produced by the needle itself, and ignores the shape of the needle-tip. These approximations are reasonable for estimating the asymptotic fields of thin needles with blunt tips. It is found that the effective Burgers vector of one needle domain is a function of spontaneous strain and the width of needle domain. From the classical theory of dislocations, the energy of the dislocation is proportional to $b^{2}$ and consequently the needle energy is proportional $w^{2}$. Widely separated fine needles thus represent a lower energy state than a single coarse needle of the same total width, provided the domain wall energy itself is negligible. Barium titanate crystals commonly form arrays of fine needles with a needle width that balances domain wall energy with the strain energy and dielectric energy due to the needle-tips.

Dislocations in a piezoelectric crystal are a source of both elastic strain and electrical polarization, due to coupling effects [29, 30]. In the needle domain, the abrupt change of polarization state near the needle-tip produces an electrical incompatibility, and each needle-tip is thus a local source of electric field which can be modelled as the field due to a distribution of equivalent charge spread over the needle-tip, as illustrated in figure 2, For a thin, blunt needle of polarization ${ }_{145} \mathbf{P}^{2}$ in a parent domain of polarization $\mathbf{P}^{1}$, the field may be approximated using a single effective line charge, $q$, given by

$$
q=\int\left(\mathbf{P}^{2}-\mathbf{P}^{1}\right) \cdot \mathbf{n} d l
$$


where, $\mathbf{n}$ is the outward normal vector of the domain wall at points on the the needle-tip. The integral in equation 4 is conducted along the domain wall, around the needle-tip. For the $90^{\circ}$ a-a needle, the resulting magnitude of effecelectric potential gradients $\varphi_{, m}$ are

$$
\begin{aligned}
\sigma_{i j} & =C_{i j k m} u_{k, m}+e_{m i j} \varphi_{, m} \\
D_{i} & =e_{i k m} u_{k, m}-\kappa_{i m} \varphi_{, m}
\end{aligned}
$$

where $C, e$ and $\kappa$ are the elastic, piezoelectric and dielectric moduli, respectively. 
Additionally, the equilibrium equations, in the absence of body forces and free charge are given by

$$
\begin{aligned}
\sigma_{i j, i} & =0 \\
D_{i, i} & =0
\end{aligned}
$$

Barnett and Lothe 31] derived the electromechanical fields and energy of an infinite straight dislocation with generalized Burgers vector $\mathbf{b}$ and generalized force vector q. The four elements of the generalized Burgers vector consist of the conventional Burgers vector (three elements) plus one element representing jump in electric potential at the dislocation path cut, which in the present work will always be zero. Similarly, the generalized force vector includes three elements of a conventional body force vector plus one element to represent a line charge density uniformly distributed over the dislocation. In the present work, the body force will be zero, but the line charge is used to produce electric fields equivalent to those caused by the polarization discontinuity at the needle-tip. The generalized displacement gradient at point $\mathbf{x}$ is then provided by 31 .

$$
\begin{aligned}
u_{I, m}= & (2 \pi|\mathbf{x}|)^{-1}\left\{-m_{m}\left(S_{I S} b_{S}+Q_{I S} q_{S}\right)+n_{m}(n n)_{I J}^{-1}\right. \\
& {\left.\left[\left(4 \pi B_{J S}+(n m)_{J K} S_{K S}\right) b_{S}+\left(S_{S J}+(n m)_{J K} Q_{K S}\right) q_{S}\right]\right\} }
\end{aligned}
$$

where lower-case indices run from 1 to 3 while upper-case indices run from 1 to 4 , such that $u_{I}(I=1 \ldots 3)$ is the displacement vector and $u_{4}$ is the electric potential. The $4 \times 4$ matrices $\mathbf{S}, \mathbf{Q}$ and $\mathbf{B}$ are related to the material constants, see Appendix A. Plane basis vectors $\mathbf{m}$ and $\mathbf{n}$ are defined such that $\mathbf{m} \cdot \mathbf{x}=|\mathbf{x}|$ and $\mathbf{n} \cdot \mathbf{x}=0$. For later use, define an out-of plane unit vector $\mathbf{k}=\mathbf{m} \times \mathbf{n}$. The components of the $4 \times 4$ matrices $(\mathbf{n n})$ and $(\mathbf{n m})$ are defined by

$$
(a b)_{J K}=a_{i} E_{i J K m} b_{m}
$$

for any vectors $\mathbf{a}$ and $\mathbf{b}$. The coefficients $E_{i J K m}$ are formed from the material 
constants as follows:

$$
\begin{aligned}
E_{i J K m} & =C_{i J K m}(J, K=1,2,3) \\
& =e_{m i J}(J=1,2,3 ; K=4) \\
& =e_{i K m}(J=4 ; K=1,2,3) \\
& =-\kappa_{i m}(J=K=4)
\end{aligned}
$$

$$
U_{t o t}=\frac{1}{2} \int_{V}\left(\sigma_{i j} u_{i, j}-D_{i} \varphi_{, i}\right) d V
$$

Defining a core region of radius $r_{0}$, the energy outside the core, in a cylindrical region of outer radius $R$ is given by 31

$$
U=\frac{1}{4 \pi}\left(4 \pi b_{J} B_{J S} b_{S}+2 q_{J} S_{J S} b_{S}+q_{J} Q_{J S} q_{S}\right) \ln \left(\frac{R}{r_{0}}\right)
$$

The Peach-Koehler force is defined to describe the mechanical force experienced by interacting dislocations. In this work, a modified Peach-Koehler force is used to quantify the interaction force on needle domains, noting that the tips of the needle domains are modelled as the combination of an effective edge dislocation coupled with an effective line charge. The modified Peach-Koehler force between two needles $N_{i}$ and $N_{j}$ comprises a mechanical force and an electrical force and is given by

$$
\mathbf{F}_{i \rightarrow j}=\left(\mathbf{b}^{j} \cdot \boldsymbol{\sigma}^{i}\right) \times \mathbf{k}+q^{j} \mathbf{E}^{i}
$$

where, $\mathbf{F}_{i \rightarrow j}$ represents the force acting on $N_{j}$ due to $N_{i}$. The needle domain $N_{j}$ is modelled as an edge dislocation $\mathbf{b}^{j}$ coupled with a line charge $q^{j}$. Accordingly, the stress $\boldsymbol{\sigma}^{i}$ and electric $\mathbf{E}^{i}$ fields are those due to the presence of needle domain $N_{i}$ in an otherwise perfect parent domain. Based on the formulation of Lubarda 
et al. 32, the existence of each needle domain produces stresses and electric fields that can be added by superposition. Thus the resultant force acting on each needle-tip is the superposition of the interaction forces due to the effect of all other needles excluding itself.

Typically, the motion of needle domains interacting with each other or responding to external loads is by extension or retraction along their length, without sideways motion or broadening. This is loosely analagous to dislocations gliding on a slip-plane without climb or change of Burgers vector. Thus the extension force, $F_{e}$, resolved along the longitudinal direction of a given needle, is:

$$
F_{e}=\mathbf{F} \cdot \mathbf{n}_{t i p}
$$

where, $\mathbf{n}_{t i p}$ is the unit vector in the extension direction. A positive extension

225 force acts to lengthen the needle domain, and a negative extension force acts to shorten the needle domain.

For analysis of the motion of interacting needle domains, a simple linear viscous model has been adopted, whereby the tip velocity is proportional to the driving force for extension. Such simple kinetics are not intended to represent accurately the temporal evolution of a domain pattern, but rather to assist computationally in relaxing the state towards equilibrium. Similar methods are commonly used for simulating dislocation dynamics 33. The velocity $v^{i}$ of the $i$ th needle domain tip is then

$$
v^{i}=\frac{1}{\eta} \sum_{j} \mathbf{F}_{j \rightarrow i} \cdot \mathbf{n}_{t i p}
$$

where, $\eta$ is a positive, viscosity-like drag coefficient. It is then of interest to study the local stability of patterns of needle domains and their evolution towards equilibrium by extension or retraction. In the present work, only extension or retraction of an existing family of needle domains is considered. Further rules would be needed to account for broadening of needles, nucleation of new needles, or annihilation, in situations where these phenomena occur. The interaction between moving needle-tips and static domain walls or free surfaces is 
also neglected: thus the domains are taken to be embedded in a crystal plate of sufficient size that edge effects do not influence the domains and where needle domain crossings do not occur. These conditions may appear at first sight very restrictive, but they are consistent with some commonly observed configurations, such as sparsely distributed needle domains terminating well within a crytal plate, or combs of parallel needle domains.

\section{Interaction of needle domains}

\subsection{Parallel domain needles with identical tips}

A configuration of two similar, parallel needle domains, labelled $N_{1}$ and $N_{2}$, is illustrated in figure 4(a). The width of each needle domain was taken to be $w=10 \mu \mathrm{m}$ for the purpose of calculation. The polarization vectors of the parent domain and needle domains are $\mathbf{P}^{1}=\left[\begin{array}{lll}0 & 1 & 0\end{array}\right] p_{0}$ and $\mathbf{P}^{N_{1}}=\mathbf{P}^{N_{2}}=[-$ $\begin{array}{lll}1 & 0 & 0\end{array} p_{0}$, respectively. Each needle can be represented by a dislocation with generalized Burgers vector $\mathbf{b}$ along $\left[\begin{array}{llll}1 & 1 & 0 & 0\end{array}\right]$ and a positive line charge $q$. The needle domain $N_{1}$ is oriented at $45^{\circ}$ to the $x_{1}$ positive direction, with its tip fixed at the origin of the $x_{1}-x_{2}$ co-ordinate system. The similar needle domain $N_{2}$ is introduced at position $\left(x_{1}, x_{2}\right)$ into the field of $N_{1}$. The interaction force on $N_{2}$ can be calculated using equations 14 and 15. A contour map of the extension force $F_{e}\left(x_{1}, x_{2}\right)$ on the needle $N_{2}$ is shown in figure $4(\mathrm{~b})$, normalized by $\sigma_{0} w$. From this figure, note that there is a line of positions at which $F_{e}=0$; this line lies close to the $x_{1}$ axis, on a line $-0.03(\mathrm{rad})$ to the positive direction of $x_{1}$ axis. When the $N_{2}$ needle-tip is located above this line, the extension force on the $N_{2}$ needle is negative but when the $N_{2}$ needle-tip is below the line, the extension force becomes positive. Thus the $N_{2}$ needle-tip is driven away from the equilibrium positions near the $x_{1}$ axis, and the line $F_{e}=0$ shows positions of unstable equilibrium. It is of interest that there appear to be no stable equilibrium positions for the pair of parallel needles.

From an energetic point of view, the absence of a stable equilibrium is illustrated using a configuration of two parallel needle domains $\left(N_{1} \& N_{2}\right)$ with 
(a)

(b)
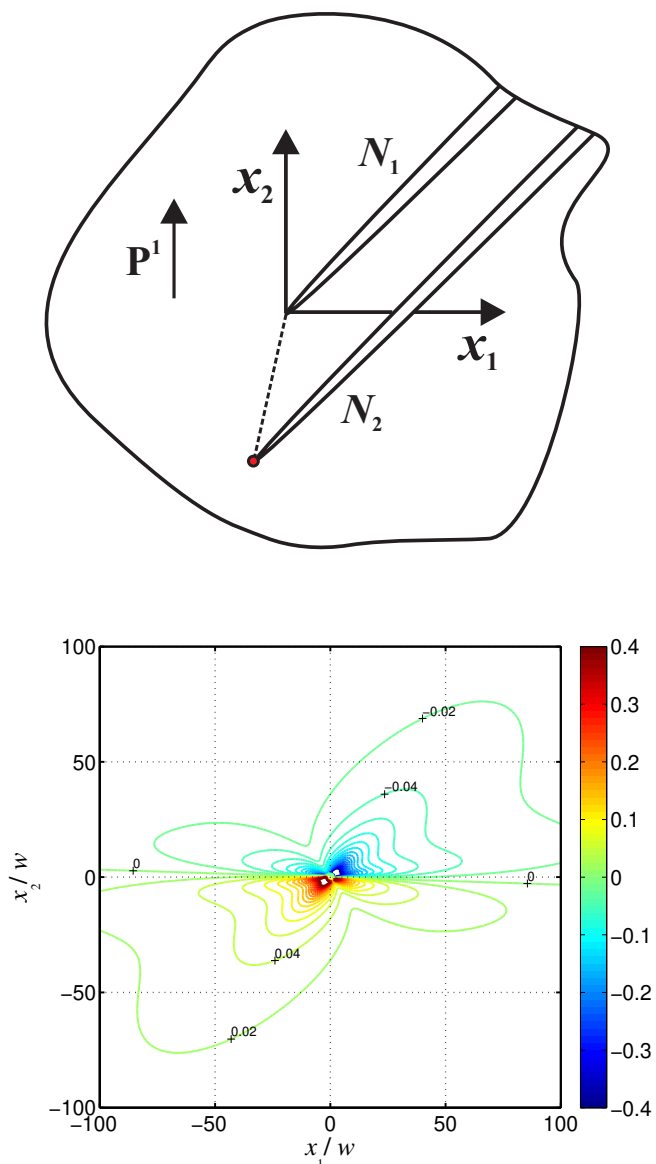

Figure 4: (a) Model configuration: a region of crystal containing two parallel needle domains with identical tips. (b) Contour map of extension force on the $N_{2}$ domain needle-tip.

spacing $s=10 \mathrm{w}$ along the $x_{1}$ axis, similar to figure $4(\mathrm{a})$. The $N_{1}$ needle domain tip is fixed at the origin of the $x_{1}-x_{2}$ co-ordinate system, while the $N_{2}$ needle-tip can be located anywhere along the line in which the needle domain lies, keeping spacing $s$ along the $x_{1}$ axis. Figure 5 show the normalized energy $U / U_{1}$ as a function of the normalized extension distance $d / w$ from the crossing point of the needle domain $N_{2}$ and the $x_{1}$ axis. Here $U$ is the total internal energy due to the two needle domains, and $U_{1}$ represents the energy produced by a single needle 


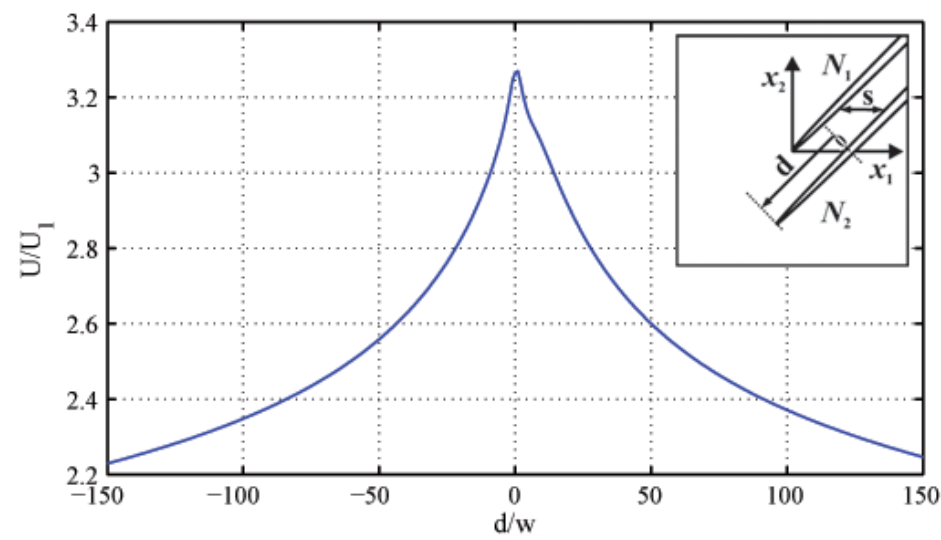

Figure 5: Normalized energy for two parallel domains with identical tips.

domain alone. The energies were computed approximately using the integral of equation 12 over a square region of side $800 w$. A square core region $(2 w \times 2 w)$ around each needle-tip was excluded from the calculation. From Figure 5 it is evident that the equilibrium position represents an unstable (maximum energy) state. Effectively, the energetic interaction is dominated by the similarly signed line charges which produce electric fields around each needle-tip such that they repel one another.

The presence of similarly signed effective line charges is based on the assumption of insulating behaviour: relaxing this assumption would allow the formation of a space charge distribution to partially screen the electric field component of the needle-tip fields. Since comb-like arrays of parallel needle domains are in practice observed in barium titanate [34, 35, the absence of stable equilibrium positions for parallel needle domains in an insulating medium leads us to explore the hypothesis that the effect of the polarization jump at the needle-tip may be partially offset by a redistribution of charge around the needle-tip.

\subsection{Stability of needle domain pairs with reduced effective line charge}

Since $\mathrm{BaTiO}_{3}$ is a semiconductor, the strong electric fields near a domain needle-tip are expected to promote local conduction, leading to a redistribution of charge, and partially screening the effect of the polarization jump. This 
charge screening provides an essential contribution to the local fields [36, 23. The effective line charge can be reduced by this process and this may give rise to a stable equilibrium. Forsbergh [6] observed that a surface polarization charge existing at a needle domain tip could be neutralized by electrical conduction.

To study the interaction of needle domains with a reduced line charge, the configuration of figure 4 was simulated and the line charge was varied in the range $Q=0$ to $Q=q=\sqrt{2} p_{0} w$. All other conditions were kept the same as in section 3.1. The results show that the existence and position of stable equilibrium states are very sensitive to changes in the quantity of effective line charge. Figure 6 presents selected contour maps of the extension force on needle $N_{2}$ with reduced effective line charge. For the two cases of full charge $Q=q$ and $Q=0.8 q$, there is no stable equilibrium position for the pair of needle domains with identical tips: the electrostatic force is strong enough to prevent the needle-tips attracting each other. When the charge is reduced to $0.73 q$, a stable equilibrium position appears. In figure 6, lines of stable equilibrium positions are marked "s" where present. The angle $\theta$ between the line of stable equilibria and the $x_{1}$ axis varies between $44.5^{\circ}$ and $58.6^{\circ}$.

Based on the above analysis of the sensitivity of the equilibrium configuration to effective line charge, the total energy of two parallel needle domains in an 315 equilibrium state was calculated, as a function of the effective line charge. The internal energies $U$ are estimated by integrating over a square region of side $200 w$ without the core region $(2 w \times 2 w)$ around each needle-tip. The needle domain $N_{1}$ is fixed at the center of this region, while the similar needle domain $N_{2}$ is spaced $10 w$ away from $N_{1}$ along the $x_{1}$ axis, with its $x_{2}$ co-ordinate chosen to provide an equilibrium configuration. The results, shown in figure 7(a) give the total internal energy, normalized by the reference energy $U_{1}$ due to a single needle domain with the full line charge $q$. Figure 7 (b) shows the corresponding angle $\theta$ between the line of equilibrium positions and the $x_{1}$ axis.

Figure 7 indicates that the energy due to two interacting needle domains 325 with reduced line charge can be greater than twice the energy of an individual needle domain with full polarization charge. The internal energy shows a 

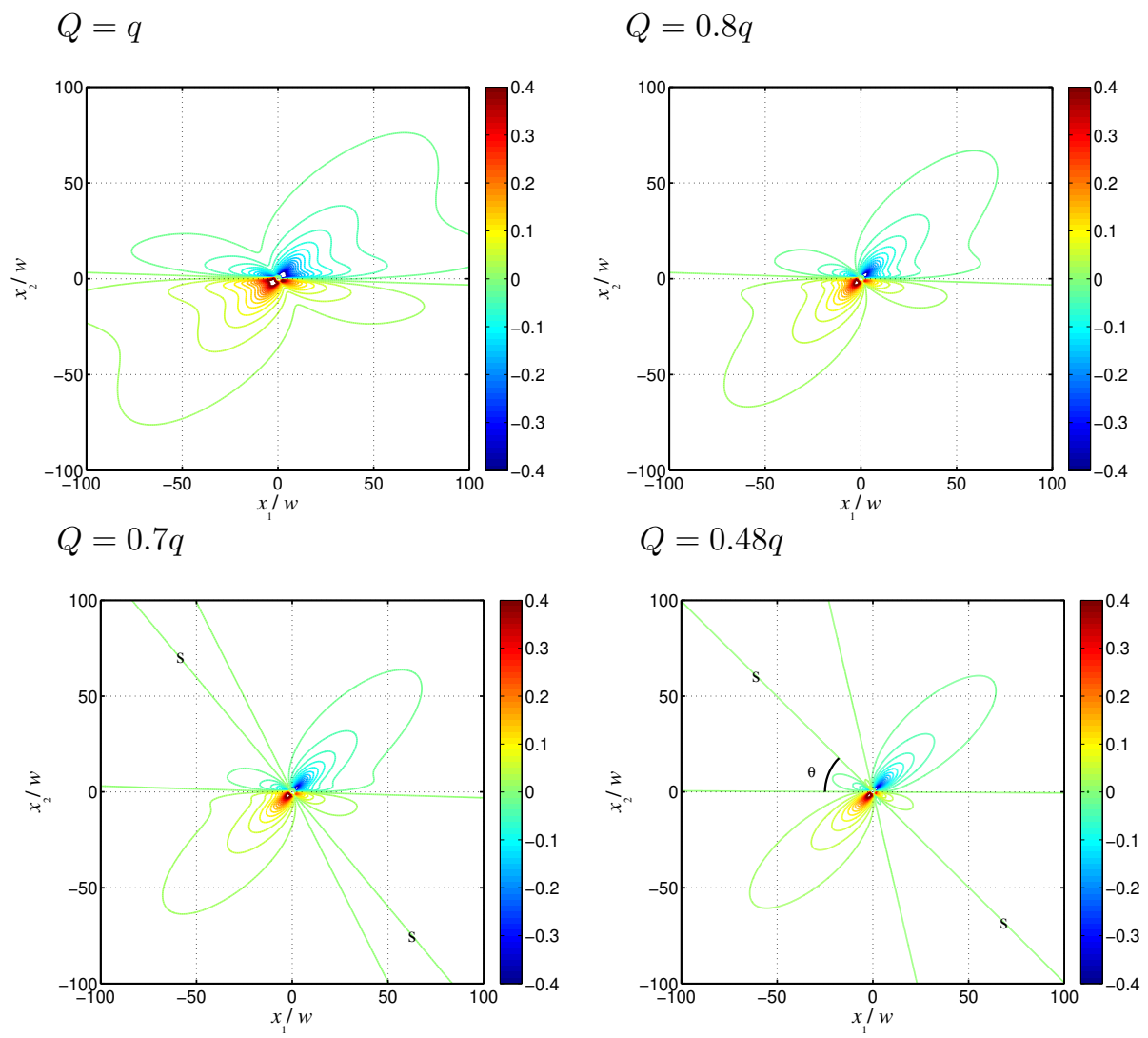

$$
Q=0.1 q
$$

$$
Q=0
$$
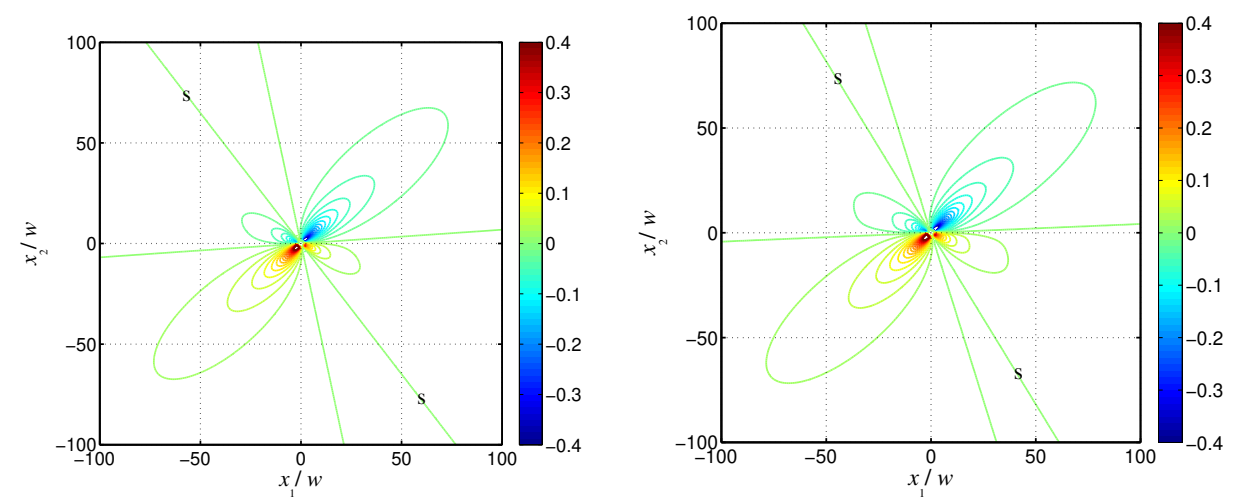

Figure 6: Contour maps of extension force on domain needle-tip $N_{2}$ for two parallel domains with identical tips, and varying line charge density from 0 to $\sqrt{2} p_{0} w$ 
(a)

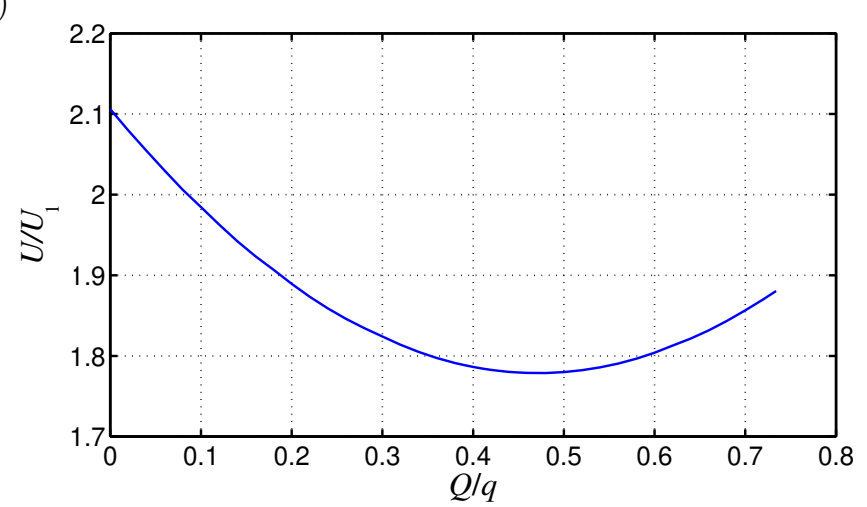

(b)

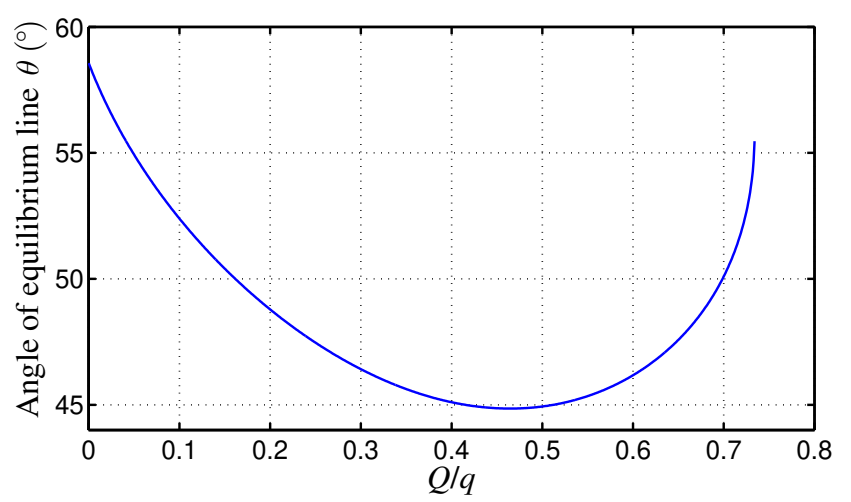

Figure 7: (a) Variation of the total internal energy due to a pair of parallel needles in an equilibrium configuration, with change in the effective line charge $Q$. (b) Variation of the stable equilibrium position (angle $\theta$ relative to the $x_{1}$ axis) with change in the effective line charge $Q$.

minimum when the equivalent line charge is about $0.48 q$. It is expected that this configuration is most stable: redistrbution of charge around the needle-tips would lead to an increase in energy, as would any relative movement of the needle-tips. Remarkably, the most stable state appears to correspond to alignment of the needle-tips on a line very close to $45^{\circ}$ to the crystallogoraphic [100] and [010] axes, a configuration observed experimentally, seen figure 1] This appears to explain the geometry of the commonly seen comb arrangement of needle domains occurring despite anisotropy, electromechanical coupling and the presence of an effective line charge. In all subsequent modelling, an effective 


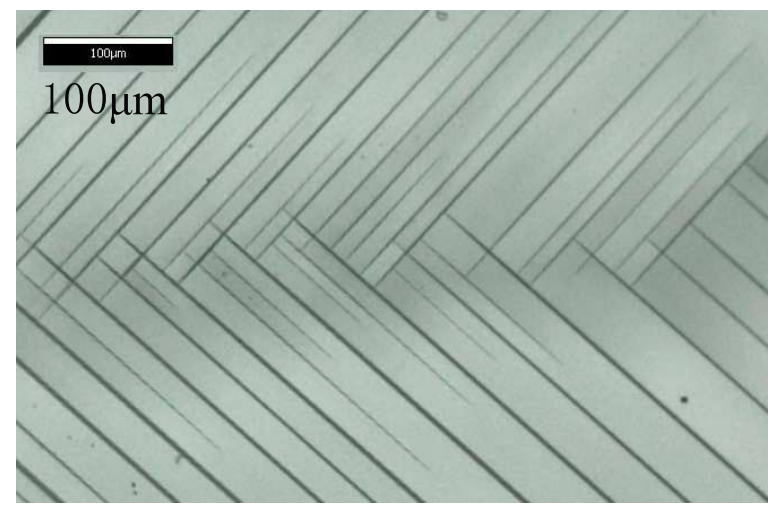

Figure 8: A herringbone array of needle domains observed by optical microscopy in a $\mathrm{BaTiO}_{3}$ crystal plate with thickness of $1 \mathrm{~mm}$.

line charge of $0.48 q$ is assumed.

\section{Herringbone pattern of needle domains}

Another characteristic pattern of needle domains is shown in figure 8 . Here the needles run perpendicular to each other, in two arrays terminating close to a line aligned with the [100] crystallographic axis. The sample thickness was $1 \mathrm{~mm}$ and the spacing of the needles is approximately $50 \mu \mathrm{m}$. Similar observations were reported by Matsumoto et al. [37] using TEM in a $\mathrm{BaTiO}_{3}$ thin film; in that case the needle spacing was approximately $300 \mathrm{~nm}$. Consider first the interaction of a pair of perpendicular needle domains as shown in figure 9(a).

The $N_{1}$ needle-tip is again located at the origin of the $x_{1}-x_{2}$ co-ordinate system, while the needle $N_{2}$ needle-tip is located at position $\left(x_{1}, x_{2}\right)$. The parent domain has polarization $\mathbf{P}^{1}=\left[\begin{array}{lll}0 & 1 & 0\end{array}\right]^{T} p_{0}$ and needle $N_{1}$ has polarization $\mathbf{P}^{N_{1}}=\left[\begin{array}{ll}-1 & 0\end{array}\right.$ $0]^{T} p_{0}$. Meanwhile, needle $N_{2}$ has an opposite polarization $\mathbf{P}^{N_{2}}=\left[\begin{array}{lll}1 & 0 & 0\end{array}\right]^{T} p_{0}$ and consequently its effective line charge has opposite sign to that of $N_{1}$.

Figure 9(b) shows a contour map of the extension force on the $N_{2}$ needle-tip. Three lines marked as $S_{1} S_{1}, S_{2} S_{2}$, and $S_{3} S_{3}$ show equilibrium positions for the $N_{2}$ needle-tip. One of these lines, $S_{2} S_{2}$, represents stable equilibrium and is oriented at $45^{\circ}$ to the $x_{1}$ axis. As this $45^{\circ}$ angle corresponds to the orientation 


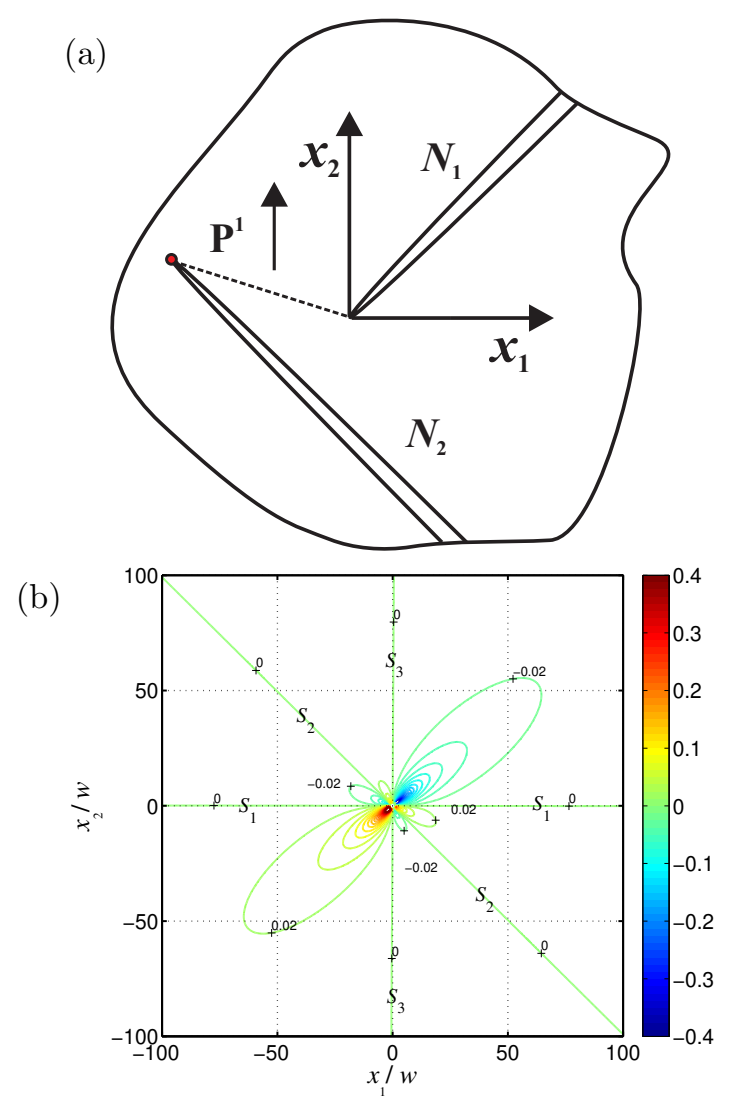

Figure 9: (a) Schematic of two perpendicular needle domains. (b) Contour map of extension force on $N_{2}$ due to the needle $N_{1}$, assuming an effective line charge of $0.48 q$.

of the $N_{2}$ needle domain, it follows that if the $N_{1}$ needle-tip lies anywhere on the line of the $N_{2}$ needle, the pair are in equilibrium. This configuration is commonly seen, wherein one needle domain appears to terminate just as it reaches another needle domain. Many such terminations can be seen in figure 8 Considering the other two lines of equilibrium positions, it is found that the $S_{3} S_{3}$ line, along the $x_{2}$ axis represents a set of unstable equilibria. If the $N_{2}$ needletip is on this line, any perturbation causing the needle $N_{2}$ to extend or shorten results in a force driving it away from the line. By contrast, the line $S_{1} S_{1}$ which lies on the $x_{1}$ axis represents stable equilibrium positions. To illustrate this, a configuration of two perpendicular needle domains with spacing $s=10 \mathrm{w}$, as 
shown in figure 10(a), was used. Let the needle $N_{2}$ changes its length so that its tip co-ordinates can vary between $(-20 w, 10 w)$ and $(10 w,-20 w)$, while the needle $N_{1}$ remains fixed at the origin. During this variation process, the needle $N_{2}$ passes through the two equilibrium lines $S_{1} S_{1}$ and $S_{3} S_{3}$. The extension force acting on each needle domain is presented in figure $10(\mathrm{~b})$. The equilibrium position $E_{1}$ is labelled; this corresponds to the state where the $N_{2}$ needle-tip 370 is situated at the stable equilibrium line $S_{1} S_{1}$. Similarly point $E_{2}$ corresponds to the unstable equilibrium with the $N_{2}$ needle-tip on line $S_{3} S_{3}$. The extension forces on needle-tip $N_{1}$ are also shown. The existence of a line of equilibria along the $x_{1}$ axis appears consistent with the observation in figure 8 where the needle-tips line up approximately along the $\left[\begin{array}{lll}1 & 0 & 0\end{array}\right]$ crystallographic axis. Locally, individual needle-tips deviate off this axis a little, drawn to terminate on the surface of other nearby needles. But globally, the needle-tips align along a $\left[\begin{array}{lll}1 & 0 & 0\end{array}\right]$ direction which defines a narrow band of low energy sites. Thus the dislocation and reduced effective charge model appears to explain well the general form of this herringbone-type array of needle domains.

Based on the above consideration of the interaction between two perpendicular needle domains, the configuration shown in figure 11(a) was modelled to give some further insight into the stability of arrays of perpendicular needles. The parent domain is again polarized along [ $\left[\begin{array}{lll}0 & 1 & 0\end{array}\right]$. Here, needle domains $1 \&$ 2 are modelled using the same effective dislocation with a positive line charge, and spaced a distance $s=10 \mathrm{w}$ along the $x_{1}$ axis direction. The needle domains $3 \& 4$ have the same spacing $(s=10 w)$, are perpendicular to domains $1 \& 2$, and have negative effective line charge. The spacing $s_{m}$ between needles 1 and 3 is half of the spacing $s$ along the $x_{1}$ axis direction.

The stability of this pattern of domains was studied by computing the variation in internal energy resulting from changing the length of each needle domain independently by an amount $d$ from the equilibrium position. As shown in figure 11(b), each needle-tip is positioned in an energy well and the configuration is thus in a stable equilibrium. It is interesting to note, however, that the needles can be pulled out of a stable configuration with relatively small extensions or 

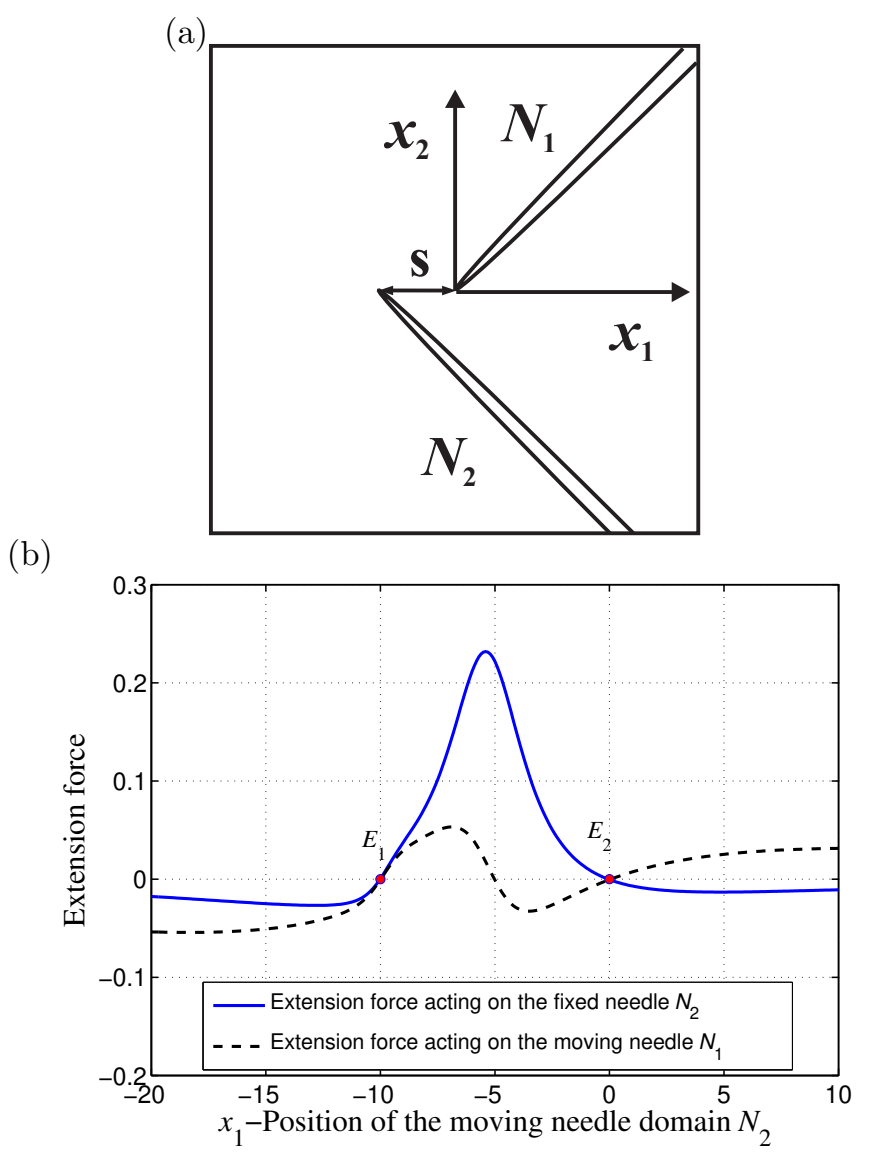

Figure 10: Interaction between two perpendicular needle domains (a) Schematic of two perpendicular needle domains with the spacing $s=10 w$; (b) The extension force acting on each needle-tip as needle $N_{2}$ extends or retracts.

retractions, on the order of a few times the needle width. The energy barrier to the escape of needle 1 by extension, or needle 4 by retraction is small (less than $1 \%$ of the total internal energy), and a displacement of less than $20 \mathrm{w}$ can remove these needles from the stable configuration. Similar calculations on a longer pattern with 30 needles indicated that there is a low energy barrier to the escape of needles towards the end of a herringbone array. Additionally, it is found that the energy well in which each needle-tip is located becomes deeper and wider when the herringbone pattern comprises an increased number of nee- 


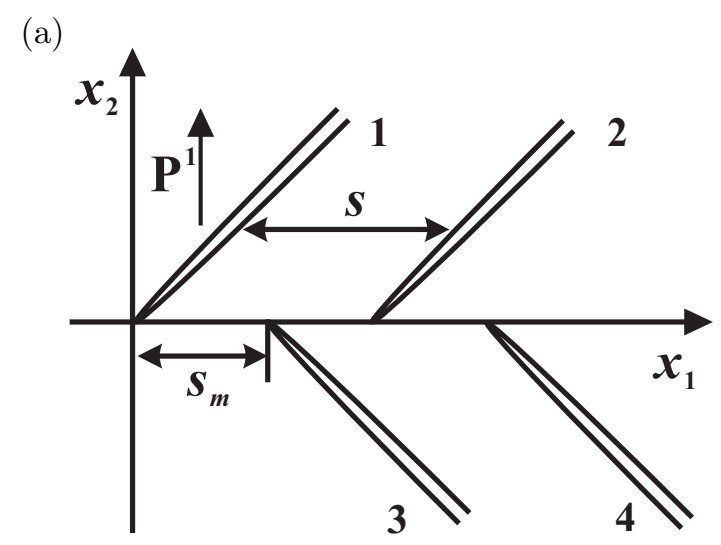

(b)

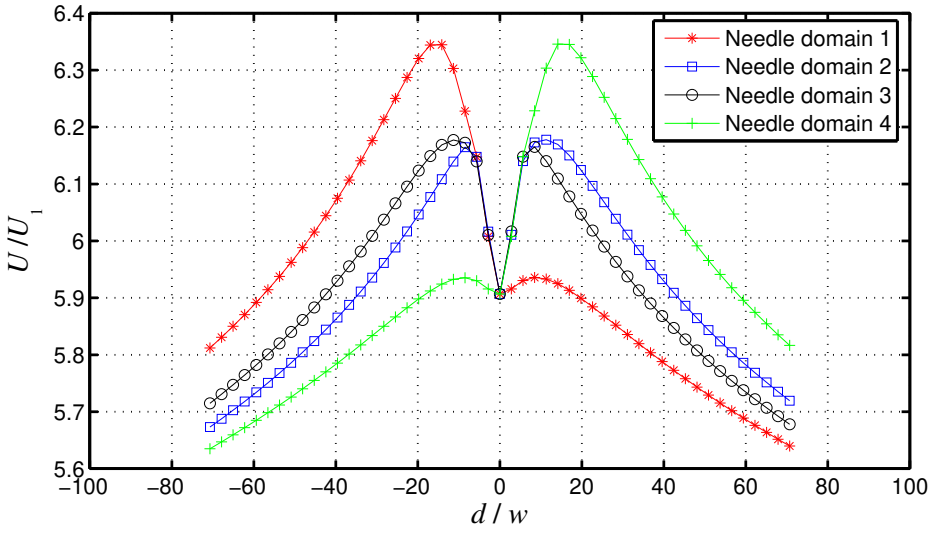

Figure 11: (a) Schematic of a short section of the herringbone pattern of needle domains; (b) Total energy variation due to an independent change in length of each needle domain.

dle domains. This means that a herringbone pattern of more needles should be more stable to perturbation, from an energetic point of view. The calculations again appear consistent with the observed herringbone-type patterns found in $\mathrm{BaTiO}_{3}$ single crystals.

\section{A needle domain detached from a comb}

What happens if a needle domain tip becomes detached from the line of needle-tips at the front of a stable comb of parallel needles? Based on the con- 

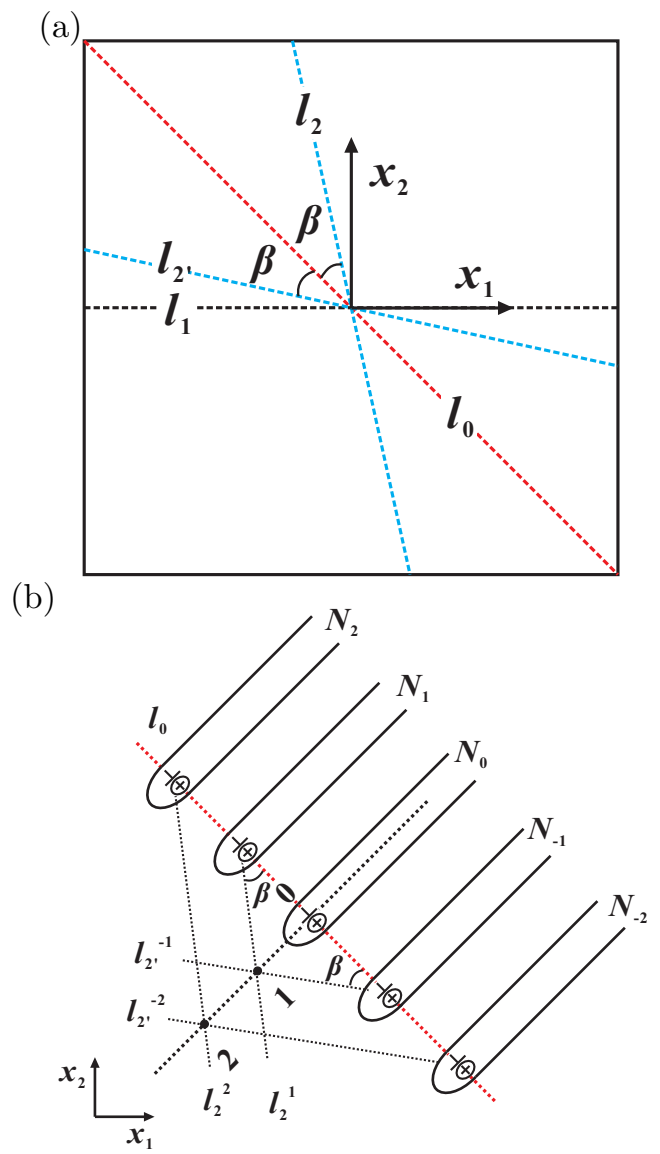

Figure 12: (a) Schematic showing lines of equilibrium positions (b) A comb of parallel needles, showing the geometry of local interactions.

tour map of interaction force between two parallel needle domains with effective line charge $Q=0.48 q$, shown in figure 6 , there are multiple lines of equilibrium positions around each needle-tip in a fixed comb. These are shown in figure 12(a). The line $l_{0}$ which is oriented at $45^{\circ}$ to the $x_{1}$ axis provides a set of stable equilibrium positions. However, the other two lines $l_{1}$ and $l_{2}$ located on the $x_{1}$ 415 axis and at $33^{\circ}$ (angle $\beta$ ) to the line $l_{0}$ represent unstable equilibrium positions. In addition, the line $l_{2^{\prime}}$ is obtained by the reflection of the line $l_{2}$ in line $l_{0}$; this will be used to explain the interaction of the detached needle domain with other nearby needles. 


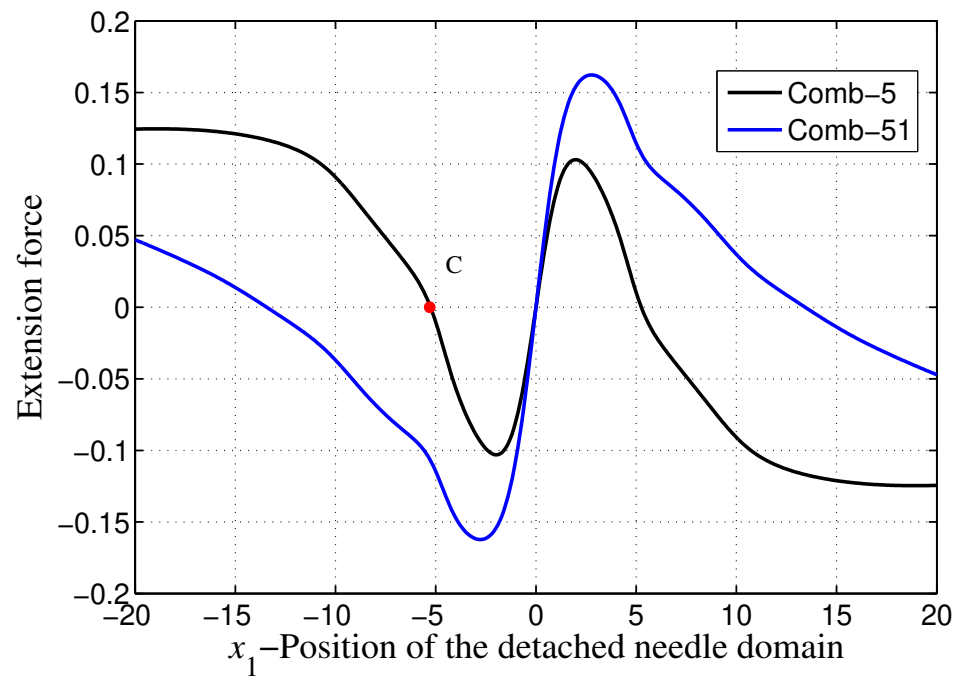

Figure 13: Extension force on a detached needle in a comb of 5 parallel needles and in a comb of 51 parallel needles. The detached needle is at the centre of the comb in each case, and negative changes in its $x_{1}$ co-ordinate represent extensions of the needle.

A comb-like configuration consisting of five identical needle domains is used to analyse the behaviour of a detached needle domain $N_{0}$, see figure 12 (b). Firstly, consider the interaction between the neighboring needle domains $N_{0}$ and $N_{1}$, with needle $N_{1}$ fixed. If needle $N_{0}$ extends away from the original equilibrium position (marked ' 0 '), the interaction energy of $N_{0}$ and $N_{1}$ will increase until the position marked ' 1 ' is reached, and decrease thereafter. Line ${ }_{425} l_{2}^{1}$ marks a line of equilibrium positions for needle $N_{0}$ in the fields of $N_{1}$. A similar interaction occurs with the needle marked $N_{-1}$. The critical position is now on the line $l_{2^{\prime}}^{-1}$. Now, the same argument applies to needles $N_{2}$ and $N_{-2}$, but the interaction energy is smaller, because of their increased distance from $N_{0}$, and the position of peak interaction energy (marked '2') is further from the line of needle-tips. In this short comb of 5 needles, the position of $N_{0}$ for maximum internal energy lies between ' 1 ' and ' 2 '. By analogy, a longer comb has a larger range between the original position ' 0 ' and the position of maximum energy for the tip of a detached needle. Figure 13 illustrates this by comparing 
the extension force on a detached needle for a short comb (5 parallel needles) and a longer comb (51 parallel needles). This figure shows the extension force of the detached needle $N_{0}$ under the effect of the whole of the rest of the comb which comprises 4 or 50 needles, respectively. For the comb of 5 needles, which is the same as the comb shown in Figure 12(b), the critical position C (an unstable equilibrium) marked in figure 13 is between positions ' 1 ' and ' 2 ', as expected. If the detached needle domain tip passes position $\mathrm{C}$, a positive extension force provided by the whole comb acts to push it further away from the line of tip fronts of the comb. The restoring force provided by a longer comb acting on a detached needle is greater than that of a short comb and acts over a longer range.

\section{Evolution of a perturbed comb of needle domains}

A regular comb-like array comprising 21 needle domains was studied. Their tips were initially located on a line orientated at $45^{\circ}$ to the $x_{1}$ axis, with spacing of $10 \mathrm{w}$ along the $x_{1}$ direction; the effective line charge at each needle-tip was set equal to $0.48 q$. What happens when this comb-like needle domain array experiences a perturbation? In the simulation, a random extension was given to each needle along its longitude direction, in the range $(-10 w / \sqrt{2},+10 w / \sqrt{2})$. This moves each needle-tip less far than position '2' of figure 12(b) from its original location. The total extension force on each needle was then obtained by superposition of the interaction forces due to all other needles. The velocity of each needle-tip was computed using equation 16 and each tip position was incremented according to the distance moved in a small, fixed time step. This process was repeated until the interaction force became zero for every needle at which point the simulation stopped in an equilibrium state. During the evolution, a sufficiently large drag coefficient $\eta$ (or equivalently, a sufficently small time step $\delta t)$ is required to avoid convergence problems. To balance the practical requirements of computing time with the requirement for convergence, the quotient $\delta t / \eta$ was set to $7 \times 10^{4} / \sigma_{0}$. Simulations were terminated when no 
step 1

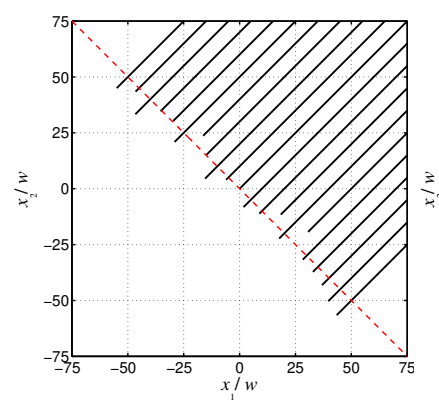

step 20

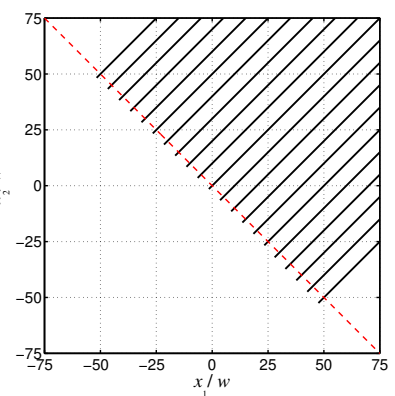

step 125

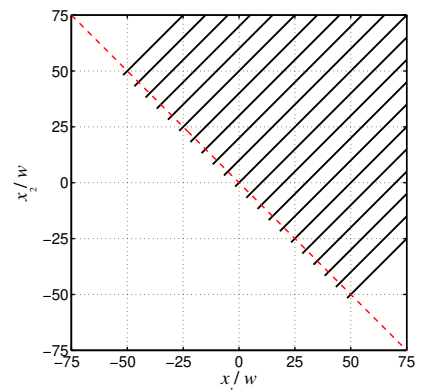

Figure 14: The evolution of the detached needle-tips under small perturbation

position increment had magnitude greater than $10^{-4} \mathrm{w}$.

Figure 14 portrays the evolution of the perturbed array of 21 needles. A dotted line is located at $x_{2}=-x_{1}$, marking the initial positions before the perturbation was applied. The needle positions after 1, 20, and 125 increments, at which point equilibrium was reached, are shown. The perturbed comb relaxes towards an equilibrium state where the needle-tips again align on a line at $45^{\circ}$ to the $x_{1}$ axis. The simulation was repeated 20 times with different random starting states, and returned to an aligned equilibrium position each time. Note that the final needle-tip positions may not coincide exactly with the initial (unperturbed) state. This can arise because the perturbations were random, so there may be a small net offset.

If the comb-like needle domains experience a larger perturbation, it may be expected that some needle-tips will escape: what will happen to them? Calculations were conducted with the same comb of 21 needles, but with perturbed initial needle lengths in the range $[-20 w / \sqrt{2},+20 w / \sqrt{2}]$. Figure 15 shows the evolution of a typical array of needle domains with this larger perturbation. In this case, it is found that the needle-tips appear to rearrange into two interspersed groups, with each group aligned along a line at $45^{\circ}$ to the $x_{1}$ axis. Some needles shorten and some extend, with the two groups appearing to repel each other. As displayed in figure 15, after 150 simulation steps the two groups have formed rather straight combs and are separated by a distance $\gg w$. They 
step 1

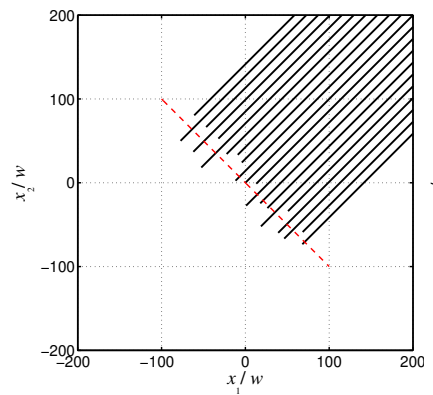

step 50

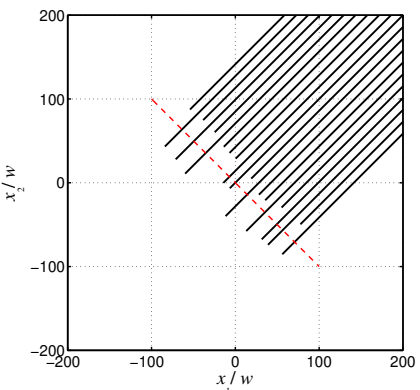

step 150

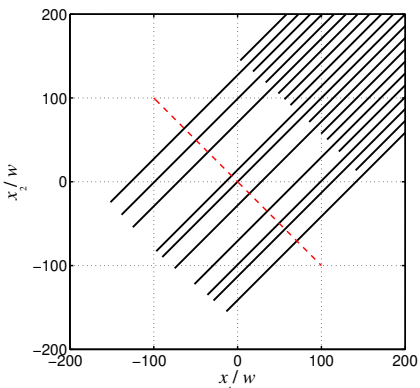

Figure 15: The evolution of the detached needle-tips under strong perturbation

have not yet reached equilibrium but now influence each other very weakly. In practice, if there is a small amount of lattice friction, this could pin such an arrangement in place. This appears consistent with experimental observations by Potnis and Huber 34] where a fine comb of needles is seen, with some detached needles loosely aligned in a coarser array, see also figure 16 .

\section{Domain bundle boundaries}

This section concerns a domain pattern consisting of $90^{\circ}$ a-a needle domains, in which parallel needles with opposite polarization states interact. First, consider the interaction between two parallel needle domains with opposite tips, as shown in figure 17. The polarization direction of the two needle domains $\left(N_{1} \& N_{2}\right)$, which are $90^{\circ}$ a-a needle domains, is along [1 100$]$ and [-1 00$]$, respectively. Here, the surrounding material forms two domains with opposite polarization. The polarization directions of the parent domains $\left(\mathrm{P}_{1} \& \mathrm{P}_{2}\right)$, whose junction forms a $180^{\circ}$ domain wall, is $\left[\begin{array}{lll}0 & -1 & 0\end{array}\right]$ and $\left[\begin{array}{lll}0 & 1 & 0\end{array}\right]$, respectively. A portion of the $180^{\circ}$ domain wall is shown as a dashed line in figure 17 The tip of the $N_{1}$ needle is located at the origin, and the needle $N_{2}$ can move relative to $N_{1}$.

Both needles lie at $45^{\circ}$ to the $x_{1}$ axis. The field of the needle $N_{1}$ in domain $\mathrm{P}_{1}$ is equivalent to that of an effective edge dislocation $\mathbf{b}_{1}=\left(\begin{array}{lll}3 & \sqrt{2} \varepsilon_{0} w / 2\end{array}\right)\left[\begin{array}{llll}1 & 1 & 0 & 0\end{array}\right]$ with a negative line charge $\mathbf{q}_{1}=-\sqrt{2} p_{0} w\left[\begin{array}{llll}0 & 0 & 0 & 1\end{array}\right]$. Correspondingly, the needle $N_{2}$ in domain $\mathrm{P}_{2}$ is approximated by an edge dislocation $\mathbf{b}_{2}=3 \sqrt{2} \varepsilon_{0} w / 2\left[\begin{array}{llll}-1 & -1 & 0 & 0\end{array}\right]$ 


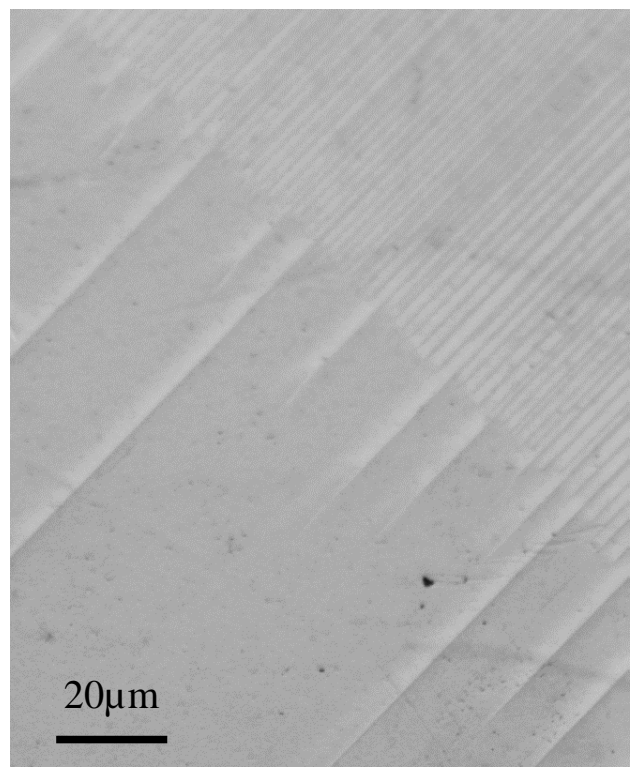

Figure 16: Optical micrograph of a $\mathrm{BaTiO}_{3}$ crystal plate showing a sparse group of needles separated from a dense comb of needle domains.

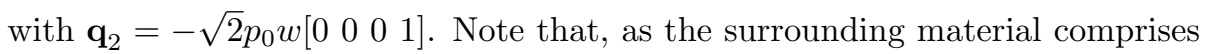
two domains, the material properties are not uniform in the region surrounding each needle. This difference causes a change in the material constants for the parent domains, dependent on the polarization direction. However, the oppositely polarized domains in $\mathrm{BaTiO}_{3}$ have identical elastic and dielectric moduli; only the piezoelectric coupling differs. Therefore the dislocation fields based on a uniform domain will not be accurate, but may still provide a crude approximation to the fields generated by these needle domains. As before, the line charge is reduced to $Q=0.48 q$ representing the minimum energy charge configuration.

After introducing the needle $N_{2}$ to the field generated by the needle $N_{1}$, interaction between of them was calculated using equations 14 and 15 A region of $100 w \times 100 w$ was simulated, and the corresponding contour map of extension 


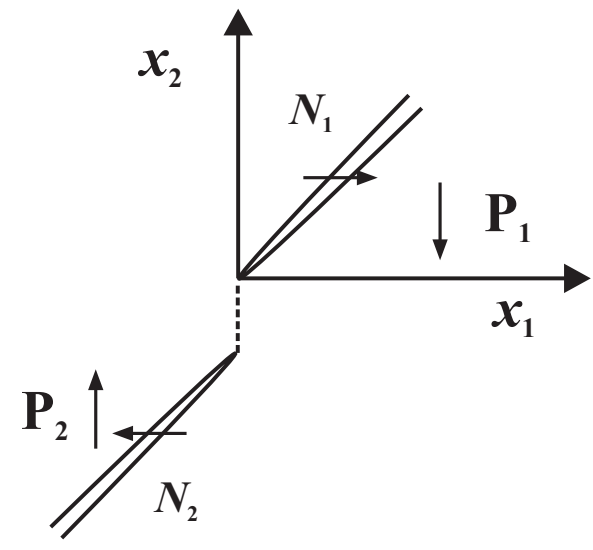

Figure 17: A pair of parallel needle domains with opposite tips.

force on $N_{2}$ is presented in figure 18(a). Three equilibrium lines a,b and c are found in this figure. The lines a and $\mathrm{c}$ are the loci of stable equilibrium states for needle $N_{2}$. Taking an example of two needle domains with spacing $20 \mathrm{w}$ along the $x_{1}$ direction, the extension force on the moving needle $N_{2}$ along its longitude direction is presented in figure 18 (b) as a function of the $x_{1}$ coordinate of the tip of $N_{2}$. The position range of needle-tip $N_{2}$ varies from $(-30 \mathrm{w},-50 \mathrm{w})$ to $(30 w, 10 w)$. In figure 18 (b), three zero-crossover points are marked by A, B, and $\mathrm{C}$ on the $x_{1}$ axis. The points $\mathrm{A}$ and $\mathrm{C}$ (which correspond to the stable equilibrium lines a and c respectively) are stable equilibrium points. However, location B is an unstable equilibrium position.

Next consider what will happen to a domain bundle composed of combs of needle domains with opposite tips in oppositely polarized parent domains. Can such an arrangement be in equilibrium? A group of six needle domains ${ }_{530} N_{1}-N_{6}$ was simulated to search for a stable equilibrium pattern. The initial arrangement is shown schematically in figure 19. In this pattern, two comb-like patterns with opposite tips are staggered, keeping the same spacing $5 w$ along the $x_{1}$ direction between each successive needle. The tips of needles $N_{1}, N_{2}, N_{3}$ are on the line $x_{2}=-x_{1}$, while the tips of needles $N_{4}, N_{5}, N_{6}$ are on the line ${ }_{535} x_{2}=-x_{1}-5 w$. As before, linear kinetics with a constant viscosity resisting 
needle-tip motion was used. Interaction forces on each needle-tip were computed by summing the contributions from the other 5 needles. The final equilibrium pattern obtained is close to the initial arrangement, as shown in figure 19(b), with only small displacements (of order $w$ ) of each needle-tip. The needle-tips align in a stable zig-zag pattern.

Experimental observations of domain bundle boundaries in $\mathrm{BaTiO}_{3}$ single crystals were made by McGilly [38] using both piezoresponse force microscopy (PFM) and scanning TEM. They found that needle domains with the opposite tips can exist in such a staggered pattern in a $\mathrm{BaTiO}_{3}$ single crystal. Addi-

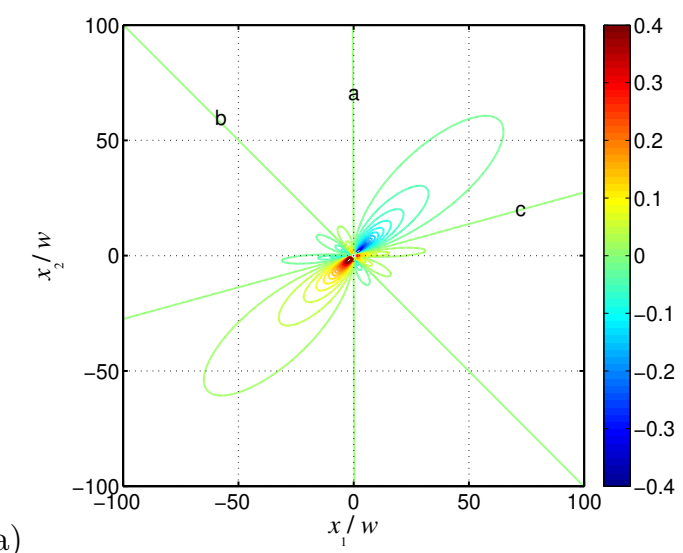

(a)

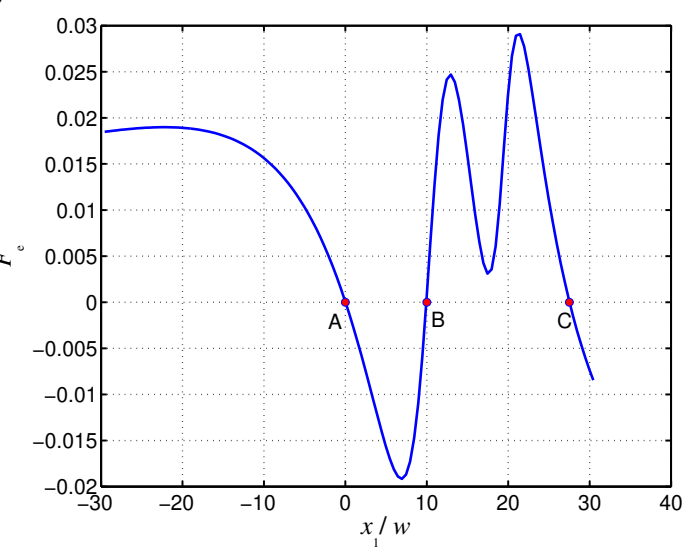

(b)

Figure 18: (a) Contour map of extension force on needle $N_{2}$; (b) extension force acting on needle $N_{2}$ moving along the line $x_{2}=x_{1}-20 \mathrm{w}$. 


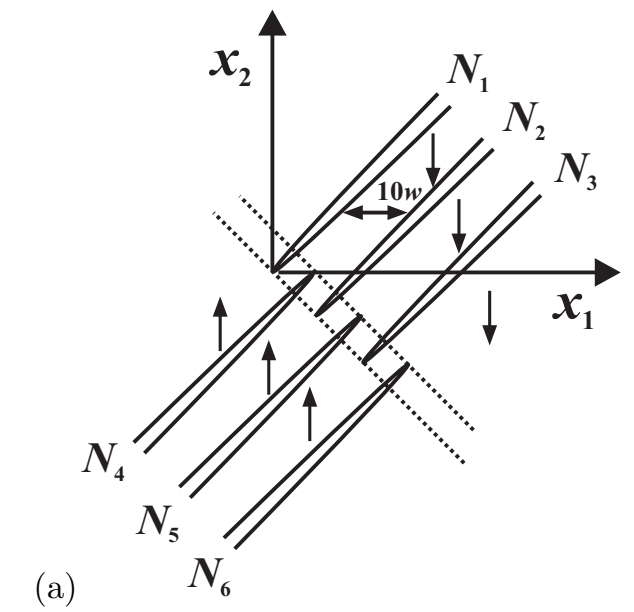

(b)

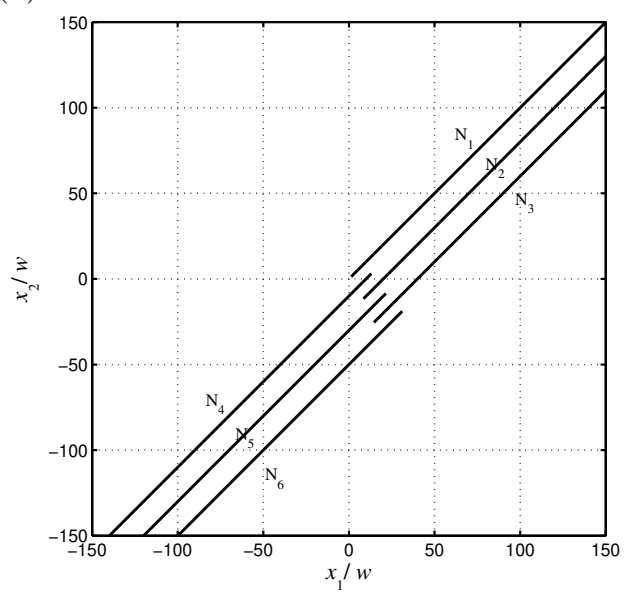

Figure 19: (a) Staggered arrangement of needle domains with opposite tips; (b) The equilibrium pattern of needle domains obtained using the dislocation model.

tionally, the zig-zag bundle boundary composed of local $180^{\circ}$ domain walls was found in their work. This observed result appears consistent with the simulation result of figure 19(b). Topological information from scanning TEM shows that domain bundle boundaries can support chains of dipole flux closure and quadrupole nanostructures. While this kind of boundary can exist in a single 550 crystal, it is comparatively rare. In the simulation, when a small perturbation (order $w$ ) disturbs one needle-tip away from equilibrium, we find that the equilibrium arrangement is restored, hence it is stable. However, strong perturbation 
(order $>10 w$ ) will cause needle-tips to escape and grow continually as they are then repelled by the other needles in the bundle. In this case, part of the nee-

dle bundle extends, while another part will shorten, resulting in the needle-tips leaving their initial positions. The dislocation and line charge model based on a uniform parent domain is a crude approximation in this case, because it neglects the variations in piezoelectric moduli in the surrounding region. Nevertheless, it appears to produce results somewhat consistent with observations of needle domain bundles.

\section{Conclusion}

The electromechanical fields produced by $90^{\circ}$ a-a needle domains in $\mathrm{BaTiO}_{3}$ single crystal plates have been modelled using the fields of an effective edge dislocation and an effective line charge. These 'fictitious' crystal defects provide a convenient means by which to study the equilibrium, interaction and evolution of needle domain patterns. Due to material anisotropy and electromechanical coupling, the interaction of needle domains differs significantly from classical results for the interaction of dislocations in isotropic media. For pairs of identical parallel needle domains in $\mathrm{BaTiO}_{3}$, no stable equilibrium configuration was found unless the effect of a screening charge distribution in reducing the effective line charge was considered.

Through studying the interaction between two needle domains with identical tips, the existence and position of stable equilibrium states were found to be sensitive to the quantity of effective line charge. When the effective line charge is reduced sufficiently, stable arrangements appeared. Remarkably, the minimum energy configuration appears to match closely to an arrangement of dislocations that is well-known in isotropic elastic media. This also appears to correspond well to experimental observations, where needle-tips align on a plane perpendicular to their longitudinal direction. Additionally, based on the study of arrays of needle domains in $\mathrm{BaTiO}_{3}$ single crystal, the model of needle domains works well to explain and analyse the stability and interaction of various 
configurations that are observed in experimental studies. This gives some confidence that the model, while approximate, may be of use in the interpretation and prediction of domain patterning in ferroelectric materials.

The work of D. Sui was supported under EPSRC grant EP/G065233/1. The authors are grateful to P. Potnis for the experimental data shown in Figure 15, and acknowledge useful discussions with Prof. C.M. Landis in the early stages of the work.

\section{Appendix A. Material matrices in the Barnett and Lothe integral} formalism

Barnett and Lothe 31] provided a convenient integral formalism using the material matrices $Q_{K S}, S_{K S}$ and $B_{K S}$ where

$$
\begin{aligned}
Q_{K S} & =-\frac{1}{2 \pi} \int_{0}^{2 \pi}(n n)_{S K}^{-1} \mathrm{~d} \theta \\
S_{K S} & =-\frac{1}{2 \pi} \int_{0}^{2 \pi}(n n)_{K J}^{-1}(n m)_{J S} \mathrm{~d} \theta \\
B_{K S} & =\frac{1}{8 \pi^{2}} \int_{0}^{2 \pi}\left((m m)_{K S}-(m n)_{K J}(n n)_{J R}^{-1}(n m)_{R S}\right) \mathrm{d} \theta
\end{aligned}
$$

with $(a b)$ defined by equation 10 and

$$
\begin{aligned}
\mathbf{m}(\theta) & =\mathbf{i} \cos \theta+\mathbf{j} \sin \theta \\
\mathbf{n}(\theta) & =-\mathbf{i} \sin \theta+\mathbf{j} \cos \theta
\end{aligned}
$$

using plane othogonal basis $(\mathbf{i}, \mathbf{j})$.

\section{References}

[1] A. K. Tagantsev, L. E. Cross, J. Fousek, Domains in ferroic crystals and thin films, Springer-Verlag New York, Inc., 2010. 
[2] D. N. Fang, Y. J. Jiang, C. T. Sun, Interactions between domain switching and crack propagation in poled $\mathrm{BaTiO}_{3}$ single crystal under mechanical loading, Acta Materialia 55 (2007) 5758-5767.

[3] W. Y. Li, C. M. Landis, Nucleation and growth of domains near crack tips in single crystal ferroelectrics, Engineering Fracture Mechanics 78 (2011) $1505-1513$.

605

[4] W. Y. Li, C. M. Landis, Modeling of the nucleation and growth of domain needles, ASME 2010 Conference on Smart Materials, Adaptive Structures and Intelligent Systems 1 (2010) 373-374.

[5] W. J. Merz, The electric and optical behavior of $\mathrm{BaTiO}_{3}$ single domain crystals, Physical Review 76 (1949) 1221-1225.

[6] P. W. Forsbergh, Domain structures and phase transitions in barium titanate, Physical Review 76 (1949) 1187-1201.

[7] J. H. Yen, Y. C. Shu, J. Shieh, J. H. Yeh, A study of electromechanical switching in ferroelectric single crystals, Journal of the Mechanics and Physics of Solids 56 (2008) 2117-2135.

[8] E. Burcsu, G. Ravichandran, K. Bhattacharya, Large electrostrictive actuation of barium titanate single crystals, Journal of the Mechanics and Physics of Solids 52 (2004) 823-846.

[9] Z. Lu, M. Xiao, Observation of localized domain reversal of iron-doped potassium niobate single crystal, Journal of Applied Physics 76 (1994) $4451-4453$.

[10] R. J. Harrison, S. A. T. Redfern, E. K. H. Salje, Dynamical excitation and anelastic relaxation of ferroelastic domain walls in $\mathrm{LaAlO}_{3}$, Physical Review B 69 (2004) 144101.

[11] J. Bornarel, J. Lajzerowicz, Experimental evidence for the long-range interaction of domains in ferroelectric $\mathrm{KH}_{2} \mathrm{PO}_{4}$, Journal of Applied Physics 39 (1968) 4339-4341. 
[12] J. Bornarel, Existence of dislocations at domain tips in ferroelectric crystal $\mathrm{KH}_{2} \mathrm{PO}_{4}$, Journal of Applied Physics 43 (1972) 845-852.

[13] J. Bornarel, J. Lajzerowicz, Interdomain and domain defect interactions in kdp, Ferroelectrics 4 (1972) 177-187.

[14] E. K. H. Salje, Y. Ishibashi, Mesoscopic structures in ferroelectric crystals: needle twins and right-angle domains, Journal of Physics: Condensed Matter 8 (1996) 8477-8495.

[15] J. F. Blackburn, E. K. H. Salje, Formation of needle shaped twin domains in cordierite: a computer simulation study, Journal of Applied Physics 85 (1999) 2414-2422.

[16] R. J. Harrison, S. A. T. Redfern, A. Buckley, E. K. H. Salje, Application of real-time, stroboscopic X-ray diffraction with dynamical mechanical analysis to characterize the motiion of ferroelectric domain walls, Journal of Applied Physics 95 (2004) 1706-1717.

[17] R. J. Harrison, E. K. H. Salje, The noise of the needle: avalanches of a single progressing needle domain in $\mathrm{LaAlO}_{3}$, Applied Physics Letters 97 (2010) 021907.

[18] E. A. Little, Dynamic behavior of domain walls in barium titanate, Physical Review 98 (1955) 978-984.

[19] J. Bornarel, J. Lajzerowicz, J. F. Legrand, Switching process in twinned ferroelectric crystals, Ferroelectrics 7 (1974) 331-314.

[20] Z. H. Zhang, X. Y. Qi, X. F. Duan, Two-step evolution mechanism of multidomains in $\mathrm{BaTiO}_{3}$ single crystal investigated by in situ transmission electron microscopy, Scripta Materialia 58 (2008) 441-444.

[21] Z. H. Zhang, X. Y. Qi, X. F. Duan, Direct determination of the polarization direction of domains in $\mathrm{BaTiO}_{3}$ single crystal, Applied Physics Letters 89 (2006) 242905. 
[22] X. Y. Qi, H. H. Liu, X. F. Duan, In situ transmission electron microscopy study of electric-field-induced $90^{\circ}$ domain switching in $\mathrm{BaTiO}_{3}$ single crystals, Applied Physics Letters 89 (2006) 092908.

[23] V. Y. Schur, A. R. Akhmatkhanov, Domain shape instabilities and dendrite domain growth in uniaxial ferroelectrics, Philosophical Transactions of the Royal Society A 376 (2018) 20170204.

[24] D. Carka, C. M. Landis, Equilibrium conditions and evolution of needle domain arrays in ferroelectric single crystals, ASME 2012 Conference on Smart Materials, Adaptive Structures and Intelligent Systems SMASIS2012-8193 (2013) 255-257.

[25] J. Agar, et al., Highly mobile ferroelastic domain walls in compositionally graded ferroelectric thin films, Nature Materials 15 (2016) 549-556.

[26] L. V. Lich, V.-H. Dinh, Formation of polarization needle-like domain and its unusual switching in compositionally graded ferroelectric thin films: an improved phase field model, RSC Advances 9 (2019) 7575-7586.

[27] Z. Li, S. K. Chan, M. H. Grimsditch, E. S. Zouboulis, The elastic and electromechanical properties of tetragonal $\mathrm{BaTiO}_{3}$ single crystals, Journal of Applied Physics 70 (1991) 7327-7332.

[28] K. Bhattacharya, G. Ravichandran, Ferroelectric perovskites for electromechanical actuation, Acta Materialia 51 (2003) 5941-5960.

[29] A. R. Hutson, L. R. Walker, Piezoelectric potentials of dislocations in insulating crystals, Journal of Applied Physics 50 (1979) 6247-6250.

[30] G. Faivre, G. Saada, Dislocations in piezoelectric semiconductors, Phys. Status Solidi (b) 52 (1972) 127-140.

[31] D. M. Barnett, J. Lothe, Dislocations and line charges in anisotropic piezoelectric insulators, Physica Status Solidi (b) 67 (1975) 105-111. 
[32] V. A. Lubarda, J. A. Blume, A. Needleman, An analysis of equilibrium dislocation distributions, Acta Metallurgica et Materialia 41 (1993) 625642 .

[33] A. Widjaja, E. Van der Giessen, V. S. Deshpande, A. Needleman, Contact area and size effects in discrete dislocation modeling of wedge indentation, Journal of Material Research 22 (2007) 655-663.

[34] P. Potnis, J. Huber, In-situ observation of needle domain evolution in barium titanate single crystals, Journal of the European Ceramic Society 33 (2013) 327-333.

[35] D. Sui, J. E. Huber, Modelling of needle domains in barium titanate single crystals using dislocation theory, Proceedings of the ASME 2010 conference on Smart Materials, Adaptive Structures and Intelligent Systems SMASIS2012-8033 (2012) 141-148.

[36] J. E. Rault, T. O. Menteş, A. Locatelli, N. Barrett, Reversible switching of in-plane polarized ferroelectric domains in $\mathrm{BaTiO}_{3}$ (001) with very low energy electrons, Scientific Reports 4 (2014) 6792.

[37] T. Matsumoto, M. Okamoto, Ferroelectric $180^{\circ}$ a-a nanostripe and nanoneedle domains in thin $\mathrm{BaTiO}_{3}$ films prepared with forcused-ion beam, IEEE Transactions on Ultrasonics, Ferroelectrics, and Frequency Control 57 (2010) 2127-2133.

700

[38] L. J. McGilly, A. Schilling, J. M. Gregg, Domain bundle boundaries in single crystal $\mathrm{BaTiO}_{3}$ lamellae: searching for naturally forming dipole flux-closure/quadrupole chains, Nano Letters 10 (2010) 4200-4205. 